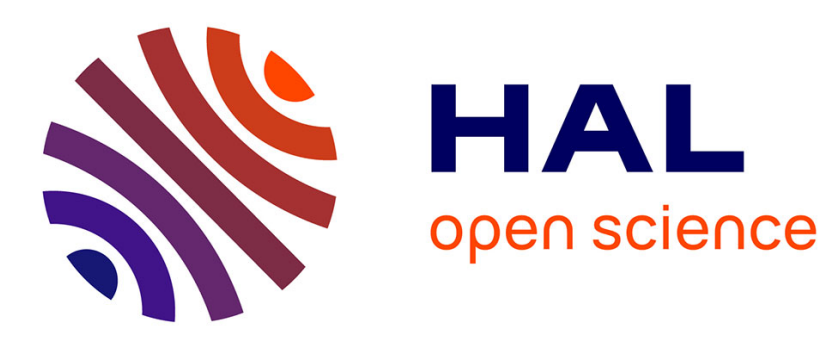

\title{
Rainwater Harvesting under Endogenous Capacity of Storage: a solution to aquifer preservation?
}

\author{
Hubert Stahn, Agnès Tomini
}

\section{To cite this version:}

Hubert Stahn, Agnès Tomini. Rainwater Harvesting under Endogenous Capacity of Storage: a solution to aquifer preservation?. 2011. halshs-00626334

\section{HAL Id: halshs-00626334 \\ https://shs.hal.science/halshs-00626334}

Preprint submitted on 26 Sep 2011

HAL is a multi-disciplinary open access archive for the deposit and dissemination of scientific research documents, whether they are published or not. The documents may come from teaching and research institutions in France or abroad, or from public or private research centers.
L'archive ouverte pluridisciplinaire HAL, est destinée au dépôt et à la diffusion de documents scientifiques de niveau recherche, publiés ou non, émanant des établissements d'enseignement et de recherche français ou étrangers, des laboratoires publics ou privés. 


\section{GREQAM}

Groupement de Recherche en Economie Quantitative d'Aix-Marseille - UMR-CNRS 6579

Ecole des Hautes études en Sciences Sociales Universités d'Aix-Marseille II et III
Document de Travail $n^{\circ}$ 2011-37

Rainwater Harvesting under Endogenous Capacity of Storage: a solution to aquifer preservation?

Hubert Stahn Agnès Tomini

September 2011 


\title{
Rainwater Harvesting under Endogenous Capacity of Storage : a solution to aquifer preservation? 出
}

\author{
Hubert Stahn * \\ GREQAM, Aix-Marseille University, Chateau LaFarge, Route des Milles, F-13290 Les Milles, FRANCE \\ Agnès Tomini \\ GREQAM, Aix-Marseille University, Chateau LaFarge, Route des Milles, F-13290 Les Milles, FRANCE
}

\begin{abstract}
This paper studies groundwater management in the presence of rainwater harvesting (RWH). We propose a two-state model that takes into account the standard dynamics of the aquifer and the dynamics of the storage capacity and we assume that the collection of rainwater reduces the natural recharge. We analyze the trade-off between these two water harvesting techniques in an optimal control model. In particular, we show that when these techniques are pure substitutes, the development of RWH leads in the long run to a depletion of the water table even if pumping is reduced.
\end{abstract}

Keywords: Rainwater Harvesting, Conjunctive Use, Groundwater Management, Optimal Control

JEL Classification: Q25, C61, D61

\section{Introduction}

Rainwater Harvesting (RWH) is a simple technique that has been used for thousands of years. Today, this practice is enjoying a revival in popularity and an international network, the International Water Association ${ }^{1}$, promotes and supports RWH initiatives worldwide as an important component in the sustainable provision of freshwater. However,

\footnotetext{
文

*Corresponding author

Email addresses: hubert.stahn@univmed.fr (Hubert Stahn ), agnes.tomini@univmed.fr (Agnès Tomini )

${ }^{1}$ See the association's website http://www.iwahq.org/home/ 
the co-existence of this technique with groundwater withdrawals is not always a success story, as it can lead to depletion of the water table.

RWH was encouraged in the early 20th century in regions with no significant river systems and remote and arid areas, sometimes on a large scale. For instance, the United Nations Environment Programme ${ }^{2}$ reports that in 2000, the Gansu province in China, which has a semi-arid to arid continental climate, reached a capacity of 2,183,000 rainwater tanks, generating 73.1 million cubic meters of drinking and supplementary irrigation water. Perrens (1982) estimates that in Australia approximately one million people rely on rainwater as their primary source of supply. Over and above these figures, this alternative supply-side measure should contribute towards a number of Millennium Development Goals, especially that of halving the proportion of the population without sustainable access to safe drinking water by 2015 (Lehmann et al., 2010). The Rainwater Partnership was established in 2004 under the initiative of the UNEP not only to promote this strategy but also to integrate it into water resources management policies. This alternative water source can therefore co-exist with other water sources, providing decision-makers with a choice between withdrawing water from the ground and investing in development of a storage technology such as cisterns or rain tanks to harvest rainwater. Our paper thus aims to extend the canonical groundwater management model to take into account the development of RWH technology.

The literature on groundwater economics is quite well-developed. Apart from the strand dealing with quality aspects, studies focus mainly on pumping patterns and strategic behaviors, based on the dynamic model proposed by Gisser and Sanchez (1980) (e.g., Gisser, 1983; Provencher, 1993; Provencher and Burt, 1993; Rubio and Casino 2003). This literature often examines the conjunctive use of two sources of water, one of which is a stock and the other a flow (Provencher, 1995). The latter is considered as uncertain and groundwater then plays a role in protecting users against this uncertainty (Tsur, 1990; Tsur and Graham-Tomasi, 1991; Knapp and Olson, 1995; Provencher and Burt, 1993).

Our work follows this tradition, with an economic model of conjunctive use of two water sources. However, we seek to determine the optimal extraction of groundwater

\footnotetext{
${ }^{2}$ See http://www.unep.or.jp/ietc/publications/urban/urbanenv-2/9.asp)
} 
in addition to the optimal level of investment in RWH facilities without uncertainty. This choice, as in the usual backstop literature ${ }^{3}$, will result from a trade-off between groundwater pumping costs and RWH investment costs. However, we are dealing with two water sources that are typically interdependent: rainwater that is harvested cannot reach the aquifer. This implies that the quantity of RWH affects the dynamics of the aquifer and even the marginal pumping cost, which depends on the aquifer head. The idea of this paper is to explore this complex dynamics and especially to show that in the long run, the introduction of RWH negatively affects the head of the aquifer.

Our analysis is based on an optimal control framework. We propose a two-state model with pure state constraints in order to combine the standard dynamics of groundwater stock and the progressive development of RWH capacity. In fact, we extend the choices available to a social planner managing an aquifer by adding the opportunity to constitute RWH capacity. The groundwater extraction part is modeled in a standard and quite general way while the constitution of RWH capacity follows the literature on capital accumulation by identifying an investment cost and a progressive depreciation of the stock of this capacity. Within this context, we specify the optimality conditions and examine the long term effects.

In a first step, we define the necessary conditions for optimality and assume that a steady state exists. This gives us the opportunity to compare the long-term groundwater level to the level that would be obtained in the same model without the opportunity to invest in RWH capacity. We essentially show that the introduction of this storage capacity leads to depletion of the aquifer. However, this result relies on the existence of a steady state. In a second step, we display that these necessary conditions are also sufficient under a standard transversality condition and that all the trajectories that satisfy these conditions admit a unique steady state, which is even a local saddle point.

This paper is organized as follows. Section 2 introduces the setting of our model. In section 3, we specify and discuss the necessary conditions for optimality. Section 4 studies the long-run effects of the development of RWH capacity. In section 5 , we focus

\footnotetext{
${ }^{3}$ For the classical backstop literature the reader is referred to Heal (1976), and Dasgupta and Heal (1979). Specific applications to groundwater resource can be found in Amigues and al. (1998), Holland and Moore (2003), Kim and al. (1989), Koundouri and Christou (2003) or Kruce and al. (1997).
} 
on the sufficient conditions in order to analyze the properties of the steady state. Section 6 provides some concluding comments.

\section{The model}

We start from a stylized dynamic and continuous time model of groundwater management. We assume that the aquifer is delineated by a flat bottom and perpendicular sides. We denote by $h(t) \in[0, \bar{h}]$ the water height where $\bar{h}>0$ stands for the maximum level of the aquifer. This water table simply rises because of recharge, which soaks through the soil to the aquifer, and falls because of water pumping ${ }^{4}$. In this stylized world, a constant quantity of RW $R>0$ is available at each $t$. Ordinarily, this quantity goes to the aquifer, but it can be stored in tanks if this storage capacity is developed. So let us denote by $w_{s}(t)$ the capacity accumulated up to time $t$. The rest of the water needs are covered by groundwater $(\mathrm{GW})$ pumping denoted by $w_{g}(t)$. In this basic case, the dynamics of the aquifer is given by:

$$
\dot{h}(t)=\left(R-w_{s}(t)\right)-w_{g}(t)
$$

The total amount of water available at time $t$ is given by $w(t)=\left(w_{g}(t)+w_{s}(t)\right)$. The instantaneous benefit induced by water use is estimated by a function $F(w)$ that behaves like a standard production function.

Assumption 1 (A1). The social benefit of the use of water is measured by a $C^{\infty}$ function $F: \mathbb{R}_{+} \rightarrow \mathbb{R}_{+}$which satisfies (i) $\forall w>0, F^{\prime}(w)>0$, (ii) $F^{\prime \prime}(w)<0$, (iii) $F(0)=0$, (iv) $\lim _{w \rightarrow 0^{+}} F^{\prime}(w)=+\infty$ and $\lim _{w \rightarrow+\infty} F^{\prime}(w)=0$

Groundwater withdrawals induce instantaneous pumping costs $C\left(w_{g}, h\right)$ depending on the amount that is pumped and on the height of the water table. As usually in this literature (Koundouri, 2004 and Rubio and Casino, 2003), we assume that $\partial_{w_{g}, h}^{2} C\left(w_{g}, h\right)<$ 0 in order to capture two basic principles related to water extraction. The first stems

\footnotetext{
${ }^{4}$ Obviously, the amount of water reaching the ground depends on various factors. One usually identifies some geological parameters like soil moisture and porosity as well as some climatic factors like evaporation or the intensity of precipitation. All these parameters define a specific hydraulic system characterized by runoffs, return flows and evaporation. However, we ignore these characteristics in order to focus on the main argument. These extensions are discussed in our concluding remarks.
} 
from the fact that when the water table is lower, it is costlier to extract water because the resource must be pumped further. In other words, the marginal extraction cost is decreasing with the water height. The second principle is related to the dynamics of the model. Using an additional unit of water, at a given period of time, lowers the water table and so raises future extraction costs. Therefore, we simply say that the marginal effect of a lowering of the water table on the future extraction cost increases with the level of pumping; this helps to slow down pumping.

Apart from this specific assumption, we also introduce usual assumptions on this cost function. This function is increasing with the level of extraction, decreasing with the height of water table and strictly convex:

Assumption 2 (A2). The groundwater extraction costs are given by a $C^{\infty}$ function $C: \mathbb{R}_{+} \times[0, \bar{h}] \rightarrow \mathbb{R}_{+}$

(i) $\forall\left(w_{g}, h\right), \partial_{w_{g}, h}^{2} C\left(w_{g}, h\right)<0$,

(ii) $\forall\left(w_{g}, h\right), \partial_{w_{g}} C\left(w_{g}, h\right)>0, \partial_{h} C\left(w_{g}, h\right)<0$ and $\partial^{2} C\left(w_{g}, h\right)$ is positive definite

The alternative water source requires an investment $I(t)$ in order to build and to maintain a storage capacity $w_{s}(t)$. This investment is made ineach period at some cost $\Theta(I)$ and the dynamics of the storage capacity takes into account an instantaneous depreciation which is measured by the function $\delta\left(w_{s}\right)$. The dynamics of the capital stock across time is therefore given by the relation:

$$
\dot{w}_{s}(t)=I(t)-\delta\left(w_{s}(t)\right)
$$

The depreciation function $\delta\left(w_{s}\right)$ and the cost function $\Theta(I)$ satisfy the following conditions:

Assumption 3 (A3). The water harvesting technique is characterized by a $C^{\infty}$ investment cost $\Theta: \mathbb{R} \rightarrow \mathbb{R}_{+}$and a $C^{\infty}$ depreciation function $\delta: \mathbb{R}_{+} \rightarrow \mathbb{R}_{+}$which respectively satisfy:

(i) $\forall I, \Theta^{\prime}(I)>0, \Theta^{\prime \prime}(I)>0$ and $\lim _{I \rightarrow 0} \Theta(I)=\lim _{I \rightarrow 0} \Theta^{\prime}(I)=0$

(ii) $\forall w_{s}, \delta^{\prime}\left(w_{s}\right)>0, \delta^{\prime \prime}\left(w_{s}\right)>0$ and $\lim _{w_{s} \rightarrow 0} \delta\left(w_{s}\right)=0$ 
Within this stylized framework, we consider the problem of a social planner who must choose the optimal GW extraction and investment path to maximize the discounted social net benefit. The discounting rate is given by $\rho>0$. We say that:

Definition 1. An optimal $G W$ extraction and investment path $\left(w_{g}^{*}(t), I^{*}(t)\right)$ solves the following control problem:

$$
\begin{aligned}
& \max _{w(t), I(t)} \int_{0}^{\infty}[\left.F\left(w_{g}(t)+w_{s}(t)\right)-C\left(w_{g}(t), h(t)\right)-\Theta(I(t))\right] e^{-\rho t} d t \\
& \text { subject to }:\left\{\begin{array}{l}
\dot{h}(t)=R-\left(w_{g}(t)+w_{s}(t)\right), \text { with } h(0)=\bar{h} \\
\dot{w}_{s}(t)=I(t)-\delta\left(w_{s}(t)\right) \text { with } w_{r}(0)=0 \\
w_{g}(t) \geq 0, h(t) \geq 0, \bar{h}-h(t) \geq 0 \\
w_{s}(t) \geq 0, R-w_{s}(t) \geq 0
\end{array}\right.
\end{aligned}
$$

We finally introduce some restrictions on our parameters $R, \bar{h}$ and $\rho$ to maintain the consistency of the model. We say that:

- If the aquifer is full $(h=\bar{h})$ and the natural recharge is extracted $\left(w_{g}=R\right)$, there is always an incentive, at least marginally, to pump an additional quantity of water even taking into account the increase in future extraction costs induced by a change in the water table (i.e. $-\frac{1}{\rho} \partial_{h} C(R ; \bar{h})$ )

- Conversely, the marginal cost of extracting one unit of water when the aquifer is empty $(h \rightarrow 0)$ is very high, at least higher than the marginal productivity of the recharge even for very small extraction levels $\left(w_{g} \rightarrow 0\right)$. This quantity is nevertheless bounded by the long-term marginal investment cost for a capacity fixed at the recharge $R$. Apart from the mathematical convenience, this last restriction simply ensures that both sources of water are always used, since it is too costly to develop a capacity that captures the whole recharge.

These assumptions can be written as:

Assumption 4 (A4). Let us assume that: 
(i) $F^{\prime}(R)>\partial_{w_{g}} C(R, \bar{h})-\frac{1}{\rho} \partial_{h} C(R, \bar{h})$

(ii) $\Theta^{\prime}(\delta(R))\left(\rho+\delta^{\prime}(R)\right)>\partial_{w_{g}} C(0,0)>F^{\prime}(R)$

\section{The Hamiltonian necessary conditions}

Let us first study the social planner problem. We are dealing with an autonomous optimal control problem with mixed and pure state constraints and we decide to adopt the direct adjoining approach (see Hartl, Sethi and Vickson, 1995). If $p_{1}(t)$ and $p_{2}(t)$ stand for the two co-state variables related respectively to the dynamics of the aquifer and of the harvesting capacity in definition 1, the present value Hamiltonian associated with this program is given $b^{5}$ :

$$
\mathcal{H}=\left[F\left(w_{g}+w_{s}\right)-C\left(w_{g}, h\right)-\Theta(I)\right]+p_{1} \cdot\left[R-\left(w_{g}+w_{s}\right)\right]+p_{2} \cdot\left[I-\delta\left(w_{s}\right)\right]
$$

It remains for us to associate the Lagrangian multipliers with each constraint. Since we have one mixed and three pure state constraints, we denote these multipliers by $\left(q_{i}(t)\right)_{i=1}^{5}$ and define the following Lagrangian:

$$
\mathcal{L}=\mathcal{H}+q_{1} \cdot w_{g}+q_{2} \cdot h+q_{3} \cdot(\bar{h}-h)+q_{4} \cdot w_{s}+q_{5} \cdot\left(R-w_{s}\right)
$$

Before going further, we first verify that::

Lemma 1. The problem given by definition 1 satisfies the constraint qualification property. Under A1(ii) and A2(ii), the Hamiltonian is also strictly concave with respect to the controls

Since the qualification constraints are verified, we can now begin to specify the necessary optimality conditions ${ }^{6}$. As usual, we know that the controls maximize the Hamiltonian along the optimal path of the state variables and verify the mixed constraints

\footnotetext{
${ }^{5}$ In order to simplify the notation, we omit the fact that all the arguments are functions of $t$ whenever possible..

${ }^{6}$ For these necessary conditions the reader is referred to Seierstad and Sydsaeter (1987) theorem 9 p.381 and note 6 p.374, Grass et al. (2008) theorem 3.60 p.149 or Hartl et al. (1995) Theorem 4.1 p186.
} 
associated with the program. Since the Hamiltonian is concave, these necessary conditions become:

$$
\left\{\begin{array}{l}
\partial_{w_{g}} \mathcal{L}=F^{\prime}\left(w_{g}+w_{s}\right)-\partial_{w_{g}} C\left(w_{g}, h\right)-p_{1}+q_{1}=0 \\
\partial_{I} \mathcal{L}=-\Theta^{\prime}(I)+p_{2}=0 \\
q_{1} \cdot w_{g}=0 \text { and } q_{1} \geq 0
\end{array}\right.
$$

Moreover, we know that the dynamics of the state and co-state variables satisfy the following conditions:

$$
\left\{\begin{array}{l}
\dot{h}=R-\left(w_{g}+w_{s}\right), \text { with } h(0)=\bar{h} \\
\dot{w}_{s}=I-\delta\left(w_{s}\right) \text { with } w_{r}(0)=0 \\
\dot{p}_{1}=\rho p_{1}-\partial_{h} \mathcal{L}=\rho p_{1}+\partial_{h} C\left(w_{g}, h\right)-q_{2}+q_{3} \\
\dot{p}_{2}=\rho p_{2}-\partial_{w_{s}} \mathcal{L}=p_{2}\left(\rho+\delta^{\prime}\left(w_{s}\right)\right)-F^{\prime}\left(w_{g}+w_{s}\right)+p_{1}-q_{4}+q_{5}
\end{array}\right.
$$

In this problem, we also have to manage pure state constraints. We therefore have the following slackness conditions:

$$
\left\{\begin{array}{lll}
q_{2} \cdot h=0, & \text { and } & q_{3} \cdot(\bar{h}-h)=0 \\
q_{4} \cdot w_{s}=0 & \text { and } & q_{5} \cdot\left(R-w_{s}\right)=0 \\
\left(q_{i}\right)_{i=2}^{5} \geq 0 &
\end{array}\right.
$$

Finally, for any entry, exit or contact time $\tau$ where the pure state constraints are binding, the co-state variables may have a discontinuity. At this junction point, there exists $\left(k_{i, \tau}\right)_{i=1}^{4} \geq 0$ with the property that:

$$
\left\{\begin{array} { l } 
{ p _ { 1 } ( \tau ^ { + } ) = p _ { 1 } ( \tau ^ { - } ) - k _ { 1 , \tau } + k _ { 2 , \tau } } \\
{ p _ { 2 } ( \tau ^ { + } ) = p _ { 2 } ( \tau ^ { - } ) - k _ { 3 , \tau } + k _ { 4 , \tau } }
\end{array} \text { and } \left\{\begin{array}{l}
k_{1, \tau} \cdot h(\tau)=0, k_{2, \tau} \cdot(\bar{h}-h(\tau))=0 \\
k_{3, \tau} \cdot w_{r}(\tau)=0, k_{4, \tau} \cdot\left(R-w_{r}(\tau)\right)=0
\end{array}\right.\right.
$$

\section{The long-term effect of RWH on the aquifer}

In this section, we highlight the fact that the introduction of RWH lowers the longterm level of the water table. This result is obtained by considering the previous necessary 
conditions and by assuming that a steady state exists. Under this assumption, we actually show that the water table and the level of groundwater extraction must verify certain relations over the long run. In a second step, we briefly recall the relations that must hold between these variables when there is no RWH. By comparing these two sets of relations, we finally conclude that the implementation of RWH depletes the aquifer.

\subsection{Some useful properties of the steady state}

We begin with the conditions that characterize our steady state. By definition, if such a point exists, the controls must be chosen in a optimal way. It follows by $(C T R)$ that:

$$
p_{1}^{*}=F^{\prime}\left(w_{s}^{*}+w_{g}^{*}\right)-\partial_{w_{g}} C\left(w_{g}^{*}, h^{*}\right)+q_{1}^{*} \text { and } p_{2}^{*}=\Theta^{\prime}\left(\delta\left(w_{s}^{*}\right)\right)
$$

Moreover, the stock variable are invariant, thus by $(D Y N)$, we have:

$$
\left\{\begin{array}{l}
R=w_{s}^{*}+w_{g}^{*}, \quad I^{*}=\delta\left(w_{s}^{*}\right) \\
p_{1}^{*}=-\frac{1}{\rho}\left(\partial_{h} C\left(w_{g}^{*}, h^{*}\right)-q_{2}^{*}+q_{3}^{*}\right) \\
p_{2}^{*}\left(\rho+\delta^{\prime}\left(w_{s}^{*}\right)\right)=F^{\prime}\left(w_{s}^{*}+w_{g}^{*}\right)-p_{1}^{*}+q_{4}^{*}-q_{5}^{*}
\end{array}\right.
$$

Finally, we also know that the slackness conditions given by $(S L C)$ are met.

Under our conjunctive use assumption, we immediately observe that the total water use $w_{s}^{*}+w_{g}^{*}$ must be equal to the recharge $R$. One of these variables can therefore be forgotten, for instance the RWH level. Moreover, if we bear in mind that system (4) defines the co-state variables, we can reduce the previous system to a set of conditions by using the two last equations of (5), which only involves the water table, the level of groundwater extraction and the Lagrangian multipliers. By adding the slackness conditions, we can therefore say that the water table and the groundwater extraction level satisfy ${ }^{7}$ :

$$
\left\{\begin{array}{l}
F^{\prime}(R)-\partial_{w_{g}} C\left(w_{g}^{*}, h^{*}\right)+\frac{1}{\rho}\left(\partial_{h} C\left(w_{g}^{*}, h^{*}\right)-q_{2}^{*}+q_{3}^{*}\right)+q_{1}^{*}=0 \\
\Theta^{\prime}\left(\delta\left(R-w_{g}^{*}\right)\right)\left(\rho+\delta^{\prime}\left(R-w_{g}^{*}\right)-\partial_{w_{g}} C\left(w_{g}^{*}, h^{*}\right)+q_{1}^{*}-q_{4}^{*}+q_{5}^{*}=0\right. \\
\text { and }\left\{\begin{array}{l}
q_{1}^{*} \cdot w_{g}^{*}=0, \quad q_{2}^{*} \cdot h^{*}=0, \quad q_{3}^{*} \cdot\left(\bar{h}-h^{*}\right)=0 \\
q_{4}^{*} \cdot\left(R-w_{g}^{*}\right)=0, \quad q_{5}^{*} \cdot w_{g}^{*}=0, \quad\left(q_{i}\right)_{i=1}^{5} \geq 0
\end{array}\right.
\end{array}\right.
$$

${ }^{7}$ The reader may be surprised by the first and the last slackness conditions. But the first follows from the non-negativity of $w_{g}^{*}$ while the last is related to the fact that $w_{s}^{*} \leq R$ (remember that $w_{g}^{*}+w_{s}^{*}=R$ ). 
In the spirit of conjunctive use, we should even go a step further and ensure that both sources of water are really used at the steady state. For instance, if no rainwater is used we are back to the standard model and this comparison makes no sense. This was exactly the motivation behind the introduction of assumption A4(ii). Since $w_{s}^{*}+w_{g}^{*}=R$, we should be able to say that:

Lemma 2. Under A2(i), A3 and $A 4(i i)$, we observe that $\left.w_{g}^{*} \in\right] 0, R[$. It follows that we can set $q_{1}^{*}=q_{4}^{*}=q_{5}^{*}=0$ in system $(6)$

Proof :Let us first assume that $w_{g}^{*}=0$. By the previous slackness conditions, we can say that $q_{4}^{*}=0$ and the second equation of $(6)$ becomes $q_{1}^{*}+q_{5}^{*}=\partial_{w_{g}} C\left(0, h^{*}\right)-\Theta^{\prime}(\delta(R))\left(\rho+\delta^{\prime}(R)\right.$. But under A2(i) and A4(ii), we observe that:

$$
q_{1}^{*}+q_{5}^{*}<\partial_{w_{g}} C(0,0)-\Theta^{\prime}(\delta(R))\left(\rho+\delta^{\prime}(R)<0\right.
$$

which contradicts the non-negativity of the multipliers. Now suppose that $w_{g}^{*}=R>0$, it follows that $q_{1}^{*}=q_{5}^{*}=0$ and,under $\mathrm{A} 3$, the second equation of $(6)$ becomes $q_{4}^{*}=-\partial_{w_{g}} C\left(R, h^{*}\right)<0$, which is a contradiction.

From all these observations, we can say that:

Remark 1. At a steady state, the water table $h^{*} \in[0, \bar{h}]$ and the groundwater extraction level $\left.w_{g}^{*} \in\right] 0, R[$ satisfy the following set of relations

$$
\left\{\begin{array}{l}
F^{\prime}(R)-\partial_{w_{g}} C\left(w_{g}^{*}, h^{*}\right)+\frac{1}{\rho}\left(\partial_{h} C\left(w_{g}^{*}, h^{*}\right)-q_{2}^{*}+q_{3}^{*}\right)=0 \\
\Theta^{\prime}\left(\delta\left(R-w_{g}^{*}\right)\right)\left(\rho+\delta^{\prime}\left(R-w_{g}^{*}\right)-\partial_{w_{g}} C\left(w_{g}^{*}, h^{*}\right)=0\right. \\
q_{2}^{*} \cdot h^{*}=0, \quad q_{3}^{*} \cdot\left(\bar{h}-h^{*}\right)=0 \text { and } q_{2}^{*}, q_{3}^{*} \geq 0
\end{array}\right.
$$




\subsection{The steady state without $R H W$}

This steady state follows from an optimal control problem which is in some sense a subcase of the problem stated in definition 1 and is given by:

$$
\begin{aligned}
& \max _{\varpi(t)} \int_{0}^{\infty}[F(\varpi(t))-C(\varpi(t), \eta(t))] e^{-\rho t} d t \\
& \text { subject to : }\left\{\begin{array}{l}
\dot{\eta}(t)=R-\varpi(t) \text { with } \eta(0)=\bar{h} \\
\varpi(t) \geq 0, \eta(t) \geq 0, \bar{h}-\eta(t) \geq 0
\end{array}\right.
\end{aligned}
$$

To avoid confusion, we denote the water table by $\eta(t)$ and the level of GW extraction by $\varpi(t)$. If we denote by $\pi$ the co-state variable and by $\left(\lambda_{i}\right)_{i=1}^{3}$ the Lagrangian multipliers associated with the three non-negativity constraints, it is immediate that the necessary conditions for optimality are simply a subset of the more general conditions in which RWH is allowed. A quick inspection of these conditions leads to the following definition of the steady state. The unique control must be optimal, the water consumption must be equal to the recharge, and $\dot{\pi}=0$.

$$
\left\{\begin{array}{l}
\pi^{*}=F^{\prime}\left(\varpi^{*}\right)-\partial_{w_{g}} C\left(\varpi^{*}, \eta^{*}\right)+\lambda_{1}^{*} \\
\varpi^{*}=R \\
\pi^{*}=\frac{1}{\rho}\left(-\partial_{\eta} C\left(\varpi^{*}, \eta^{*}\right)+\lambda_{2}^{*}-\lambda_{3}^{*}\right)
\end{array}\right.
$$

and the following slackness conditions have to hold:

$$
\lambda_{1}^{*} \cdot \varpi^{*}=0, \lambda_{2}^{*} \cdot \eta^{*}=0, \lambda_{3}^{*} \cdot\left(\bar{h}-\eta^{*}\right)=0 \text { with }\left(\lambda_{i}^{*}\right)_{i=1}^{3} \geq 0
$$

Since the extraction of groundwater $w_{g}^{*}$ must be equal to the total recharge $R$, we can say that:

Remark 2. The water table without RWH satisfies:

$$
\left\{\begin{array}{l}
F^{\prime}(R)-\partial_{w_{g}} C\left(R, \eta^{*}\right)+\frac{1}{\rho}\left(\partial_{\eta} C\left(R, \eta^{*}\right)-\lambda_{2}^{*}+\lambda_{3}^{*}\right)=0 \\
\lambda_{2}^{*} \cdot \eta^{*}=0, \lambda_{3}^{*} \cdot\left(\bar{h}-\eta^{*}\right)=0 \text { with }\left(\lambda_{i}^{*}\right)_{i=2}^{3} \geq 0
\end{array}\right.
$$

Now we can compare the water table $\eta^{*}$ that solves (9) with $h^{*}$, which solves $(7)$. 


\subsection{The effect of $R H W$}

Let us now verify that the introduction of RWH leads to a depletion of the aquifer. This result is trivial in two following cases.

Remark 3. By using the slackness conditions, we can observe that:

(i) if $q_{2}^{*}>0$, the aquifer with $R W H$ is empty, i.e. $h^{*}=0$ solves system (7). It follows trivially that $h^{*}<\eta^{*}$ unless $\eta^{*}=0$.

(ii) if $\lambda_{3}^{*}>0$, the aquifer without $R W H$ is full, i.e. $\eta^{*}=\bar{h}$ verifies system (9) and trivially $h^{*}<\eta^{*}$ unless $h^{*}=\bar{h}$

We can, in the rest of the discussion, set $q_{2}^{*}=0$ and $\lambda_{3}^{*}=0$ (7) and (9) respectively

These two remarks simplify the argument since the first equations of (7) and (9) become respectively:

$$
\left\{\begin{array}{l}
F^{\prime}(R)=\partial_{w_{g}} C\left(w_{g}^{*}, h^{*}\right)-\frac{1}{\rho} \partial_{h} C\left(w_{g}^{*}, h^{*}\right)-\frac{q_{3}^{*}}{\rho} \\
F^{\prime}(R)=\partial_{w_{g}} C\left(R, \eta^{*}\right)-\frac{1}{\rho} \partial_{\eta} C\left(R, \eta^{*}\right)+\frac{\lambda_{2}^{*}}{\rho}
\end{array}\right.
$$

Let us now recall by lemma 2 that $w_{g}^{*}<R$. If we also bear in mind that the total pumping cost, by $\mathrm{A} 2(\mathrm{ii})$, is increasing in $w_{g}$, the first equation becomes:

$$
F^{\prime}(R)<\partial_{w_{g}} C\left(R, h^{*}\right)-\frac{1}{\rho} \partial_{h} C\left(R, h^{*}\right)-\frac{q_{3}^{*}}{\rho}
$$

It follows by using the second equation that:

$$
\partial_{w_{g}} C\left(R, \eta^{*}\right)-\frac{1}{\rho} \partial_{\eta} C\left(R, \eta^{*}\right)+\frac{\lambda_{2}^{*}}{\rho}<\partial_{w_{g}} C\left(R, h^{*}\right)-\frac{1}{\rho} \partial_{h} C\left(R, h^{*}\right)-\frac{q_{3}^{*}}{\rho}
$$

Since $\lambda_{2}^{*}, q_{3}^{*} \geq 0$, this implies that:

$$
\partial_{w_{g}} C\left(R, \eta^{*}\right)-\frac{1}{\rho} \partial_{\eta} C\left(R, \eta^{*}\right)<\partial_{w_{g}} C\left(R, h^{*}\right)-\frac{1}{\rho} \partial_{h} C\left(R, h^{*}\right)
$$

Finally, we know by A2(i)(ii) that the total pumping cost is decreasing in the water table. We can therefore say that: 
Proposition 1. Under our assumptions, the introduction of $R W$ harvesting, even if it is welfare improving, leads to environmental damage to the aquifer since $h^{*}<\eta^{*}$ unless the aquifer is either empty or full.

\section{The existence of a steady state}

At this point, we know that any optimal path which solves our water management problem and admits a steady state has the property that the long-term water table is lower than that obtained without RWH. To strengthen this result, we now show that any path that satisfies the sufficient optimality conditions admits a unique steady state which is locally a saddle point. To simplify this discussion, we restrict our analysis to interior solutions. Up to now we have not used assumption A4(i), which, as we will see, ensures that the aquifer is never full or empty.

According to the Mangasarian sufficient conditions (see Hartl, Sethi and Vickson, 1995, theorem 8.4 p.203), we simply make sure that the Hamiltonian is strictly concave in the controls and the state variable while the constraints are quasi-concave in these variables. By imposing a transversality condition, we can state that:

Lemma 3. A path $\left(\tilde{w}_{g}, \tilde{I}, \tilde{w}_{r}, \tilde{h}\right)$ that satisfies CRT, DYN, SLC is an optimal path provided that:

$$
\lim _{t \rightarrow+\infty} p_{1}(t)(h(t)-\tilde{h}(t))+p_{2}(t)\left(w_{r}(t)-\tilde{w}_{r}(t)\right)=0
$$

Let us now show that a unique steady state exists. From the discussion that we have conducted in sub-section 4.1, we know that the steady state verifies system (7). Moreover if we now use assumption A4, we can verify that:

Lemma 4. Using now A4(i), the water table is never full nor empty. This means that the steady state solves:

$$
\left\{\begin{array}{l}
F^{\prime}(R)-\partial_{w_{g}} C\left(w_{g}^{*}, h^{*}\right)+\frac{1}{\rho} \partial_{h} C\left(w_{g}^{*}, h^{*}\right)=0 \\
\Theta^{\prime}\left(\delta\left(R-w_{g}^{*}\right)\right)\left(\rho+\delta^{\prime}\left(R-w_{g}^{*}\right)-\partial_{w_{g}} C\left(w_{g}^{*}, h^{*}\right)=0\right.
\end{array}\right.
$$


Proof Let us assume that the water table that solves system (7) of subsection 4.1 is respectively full or empty, and let us exhibit a contradiction on the sign of Lagrangian multipliers. So, if $h^{*}=\bar{h}$, the slackness conditions entail that $q_{2}^{*}=0$ and the first equation of system (7) becomes:

$$
\frac{1}{\rho} q_{3}^{*}=-F^{\prime}(R)+\partial_{w_{g}} C\left(w_{g}^{*}, \bar{h}\right)-\frac{1}{\rho} \partial_{d} C\left(w_{g}^{*}, \bar{h}\right)
$$

But under A2(ii), the right-hand side of this equality is increasing in $w_{g}^{*}$. Moreover, by lemma $2 w_{g}^{*}<R$ and A4(i), we obtain the following contradiction:

$$
\frac{1}{\rho} q_{3}^{*}<-F^{\prime}(R)+\partial_{w_{g}} C(R, \bar{h})-\frac{1}{\rho} \partial_{d} C(R, \bar{h})<0
$$

Now assume that $h^{*}=0$, then $q_{3}^{*}=0$ and the first equation of (7) can be written:

$$
\frac{1}{\rho} q_{2}^{*}=F^{\prime}(R)-\partial_{w_{g}} C\left(w_{g}^{*}, 0\right)+\frac{1}{\rho} \partial_{h} C\left(w_{g}^{*}, 0\right)
$$

Since $\partial_{h} C\left(w_{g}^{*}, 0\right) \leq 0$, and $\partial_{w_{g} w_{g}}^{2} C\left(w_{g}, h\right)>0, q_{2}^{*}$ is bounded from above by $\rho\left(F^{\prime}(R)-\partial_{w_{g}} C(0,0)\right)$ which is negative under A4(ii)

The question of the existence and the uniqueness of the steady state can therefore be reduced to the study of system (10). Given our general setting, the proof of these results relies on differential topology and uses a degree theory argument (see for instance Mas-Colell, 1985, p.207-08).

Proposition 2. There exists a unique interior steady state.

It remains for us to verify the stability properties of this steady state by linearizing the differential system given by (DYN). Since we have an interior equilibrium, we can set all the multipliers to zero and obtain the following system:

$$
\left(\begin{array}{l}
\dot{h} \\
\dot{w}_{s} \\
\dot{p}_{1} \\
\dot{p}_{2}
\end{array}\right)=\left[\begin{array}{cccc}
-\frac{\partial_{w_{g}, h}^{2} C}{F^{\prime \prime}-\partial_{w_{g}, w_{g}}^{2} C} & \frac{\partial_{w_{g}, w_{g} C}^{2} C}{F^{\prime \prime}-\partial_{w_{g}, w_{g}}^{2} C} & -\frac{1}{F^{\prime \prime}-\partial_{w_{g}, w_{g}}^{2} C} & 0 \\
0 & -\delta^{\prime} & 0 & \frac{1}{\delta^{\prime \prime}} \\
\frac{\left(\partial_{w_{g}, h}^{2} C\right)^{2}}{F^{\prime \prime}-\partial_{w_{g}, w_{g} C}^{2} C}+\partial_{h, h}^{2} C & -\frac{F^{\prime \prime} \cdot \partial_{w_{g}, h}^{2} C}{F^{\prime \prime}-\partial_{w_{g}, w_{g}}^{2} C} & \rho+\frac{\partial_{w_{g}, h}^{2} C}{F^{\prime \prime}-\partial_{w_{g}, w_{g} C}^{2} C} & 0 \\
-\frac{F^{\prime \prime} \cdot \partial_{w_{g}, h}^{2} C}{F^{\prime \prime}-\partial_{w_{g}, w_{g}}^{2} C} & p_{2} \cdot \delta^{\prime \prime}+\frac{F^{\prime \prime} \cdot \partial_{w_{g}, w_{g} C}^{2} C}{F^{\prime \prime}-\partial_{w_{g}, w_{g} C}^{2} C} & -\frac{\partial_{w_{g}, w_{g} C} C}{F^{\prime \prime}-\partial_{w_{g}, w_{g}}^{2} C} & \rho+\delta^{\prime}
\end{array}\right] \cdot\left(\begin{array}{c}
h-h^{*} \\
w_{s}-w_{s}^{*} \\
p_{1}-p_{1}^{*} \\
p_{2}-p_{2}^{*}
\end{array}\right)
$$

Following Dockner and Feichtinger (1991), we can show that: 
Proposition 3. This steady state is locally a saddle point

\section{Concluding remarks}

This paper extends the standard groundwater extraction model by introducing the opportunity to access a new source of water (rainwater) through investment in capital. Rainwater harvesting requires investment to build and maintain a storage and irrigation capacity that can be used jointly with groundwater. The main conclusion of this model is that the level of the aquifer, at the steady state, will be lower in the presence of this RWH capacity. We derive this result from the Hamiltonian necessary conditions of an intertemporal water management program by assuming, in a first step, the existence of a steady state. In a second step, we prove that these conditions are also sufficient, provided that a standard tranversality condition holds, and we verify that all these trajectories have a unique steady state which is locally a saddle point.

However, to isolate the effect of rainwater harvesting on groundwater extraction as well as on the level of the aquifer depth, we consider the simplest possible dynamic setting with (i) a simple "bathtub" aquifer, i.e. a flat bottom with parallel sides, (ii) the social planner approach (iii) complete information on hydrological characteristics (iv) no uncertainty on capital.

These simplifications call for future extensions. Namely, in line with the literature relaxing some Gisser-Sanchez assumptions, it could be interesting to incorporate a more accurate depiction of groundwater hydrology and rainwater variability. For instance, Brozovic et al. (2003) or Saak and Peterson (2007) integrate spatially variable features such as the speed of lateral flow or differences in the elevation of the bottom of the aquifer. Thus, in our setting, one might expect the introduction of a two-cell aquifer where the elevation of bottom differs across location to impact on our result through the trade-off based on marginal costs.

Along the same lines, one could also consider more complex dynamics including, for instance, return flows from irrigation to the aquifer, a more complex infiltration process and water evaporation during storage. This would surely mitigate our clear-cut result that the water table is always depleted. For instance, Stahn and Tomini (2010) show that there exist certain hydrological characteristics under which the impact of RWH is not negative. 
But these results are obtained in a context where RWH is a backstop technology requiring no capital accumulation.

Another more detailed characterization could lead us to incorporate uncertainties about rainfall variability, following Fisher and Rubio (1997), for instance, who model water resources as a stochastic process and focus on the determination of long-term water storage capacity. Failure to take uncertainty into account can lead to costly errors. In other words, by reckoning random capital in order to capture fluctuations in precipitations, one might expect the water level of the aquifer in the steady state to depend on risk behavior as well as the level of uncertainty.

Thus, various refinements can be applied to this model to achieve a more detailed approach. Nevertheless, the results should be fairly similar to our main finding about the impact on the aquifer water level. It is therefore interesting to wonder about the significance of this result with respect to the principle of sustainable development. Groundwater also maintains the health of the ecosystem, which gives it a conservation value. In other words, the question must be addressed of whether the implementation of this technology might not jeopardize the sustainable level of groundwater for all its different functions.

\section{APPENDIX}

\section{Appendix A. Proof of Lemma 1}

(i) The qualification constraint.

Following Grass and al. (2008) th. 3.60, we have to verify that:

$$
Q_{1}=\left[\partial_{w_{g}, I}\left(w_{g}\right), w_{g}\right] \text { and } Q_{2}=\left[\begin{array}{ccccc}
\partial_{\omega_{g}, I}\left(\partial_{h}(h) \cdot \dot{h}+\partial_{w_{r}}(h) \cdot \dot{w}_{r}\right) & h & 0 & 0 & 0 \\
\partial_{\omega_{g}, I}\left(\partial_{h}(\bar{h}-h) \cdot \dot{h}+\partial_{w_{r}}(\bar{h}-h) \cdot \dot{w}_{r}\right) & 0 & \bar{h}-h & 0 & 0 \\
\partial_{\omega_{g}, I}\left(\partial_{h}\left(w_{r}\right) \cdot \dot{h}+\partial_{w_{r}}\left(w_{r}\right) \cdot \dot{w}_{r}\right) & 0 & 0 & w_{r} & 0 \\
\partial_{\omega_{g}, I}\left(\partial_{h}\left(R-w_{r}\right) \cdot \dot{h}+\partial_{w_{r}}\left(R-w_{r}\right) \cdot \dot{w}_{r}\right) & 0 & 0 & 0 & R-w_{r}
\end{array}\right]
$$


are matrices of full rank. Moreover, it is immediate that these matrices are given by:

$$
Q_{1}=\left[\begin{array}{lll}
1 & 0 & w_{g}
\end{array}\right], Q_{2}=\left[\begin{array}{cccccc}
1 & 0 & d & 0 & 0 & 0 \\
-1 & 0 & 0 & \bar{h}-h & 0 & 0 \\
0 & 1 & 0 & 0 & w_{r} & 0 \\
0 & -1 & 0 & 0 & 0 & R-w_{r}
\end{array}\right]
$$

$Q_{1}$ is obviously of full rank. Concerning $Q_{2}$, let us remember that $\bar{h}, R>0$. This means that we can always choose a non-zero vector when we respectively consider the columns 3,4 and 5,6 . If we add to this choice the 2 first columns we can conclude that $Q_{2}$ is of rank 4 .

(ii) The concavity of the Hamiltonian with respect to the controls.

For definition 1, a simple computation shows that:

$$
\partial_{\left(w_{g}, I\right)}^{2} \mathcal{H}=\left[\begin{array}{cc}
F^{\prime \prime}\left(w_{g}+w_{s}\right)-\partial_{w_{g}, w_{g}}^{2} C\left(w_{g}, h\right) & 0 \\
0 & -\Theta^{\prime}(I)
\end{array}\right]
$$

Moreover, under A1(ii) and A2(ii), $\partial_{\left(w_{g}, I\right)}^{2} \mathcal{H}$ is negative definite.

\section{Appendix B. Proof of lemma 3}

It remains for us to verify that the Hamiltonian $\mathcal{H}\left(w_{g}, I, h, w_{r},\left(p_{i}\right)_{i=1}^{2}\right)$ is strictly concave in $\left(w_{g}, I, h, w_{r}\right)$ and that the different constraints are quasi-concave in these variables. The last condition is always satisfied since our constraints are linear. So let us now compute the Hessian of this Hamiltonian for each $\left(p_{i}\right)_{i=1}^{2}$. We obtain by taking the following order of the variables $\left(w_{g}, w_{r}, h, I\right)$

$$
\partial^{2} \mathcal{H}=\left[\begin{array}{cccc}
F "-\partial_{w_{g}, w_{g}}^{2} C & F^{\prime \prime} & -\partial_{w_{g}, h}^{2} C & 0 \\
F^{\prime} & F^{\prime \prime}-p_{2} \delta " & 0 & 0 \\
-\partial_{w_{g}, d}^{2} C & 0 & -\partial_{d, d}^{2} C & 0 \\
0 & 0 & 0 & -\Theta "
\end{array}\right]
$$

Bearing in mind that $\Theta^{\prime}(I)=p_{2}$, we observe, under assumption A1(ii), A2(i)(ii) and A3(i)(ii), that:

$$
\begin{aligned}
& D_{1}=F^{\prime \prime}-\partial_{w_{g}, w_{g}}^{2} C<0 \\
& D_{2}=\left|\begin{array}{cc}
F^{\prime \prime}-\partial_{w_{g}, w_{g}}^{2} C & F^{\prime \prime} \\
F^{\prime \prime} & F^{\prime \prime}-\Theta^{\prime} \delta^{\prime \prime}
\end{array}\right|=-F^{\prime \prime}\left(\partial_{w_{g}, w_{g}}^{2} C+\Theta^{\prime} \delta^{\prime \prime}\right)+\Theta^{\prime} \delta^{\prime \prime} \partial_{w_{g}, w_{g}}^{2} C>0
\end{aligned}
$$




$$
\begin{aligned}
& D_{3}=\left|\begin{array}{ccc}
F^{\prime \prime}-\partial_{w_{g}, w_{g}}^{2} C & F^{\prime \prime} & -\partial_{w_{g}, d}^{2} C \\
F^{\prime \prime} & F^{\prime \prime}-\Theta^{\prime} \delta " & 0 \\
-\partial_{w_{g}, h}^{2} C & 0 & -\partial_{h, h}^{2} C
\end{array}\right| \\
& =F^{\prime \prime} \Theta^{\prime} \delta^{\prime \prime} \partial_{h, h}^{2} C+\left(F^{\prime \prime}-\Theta^{\prime} \delta^{\prime \prime}\right)\left(\partial_{w_{g}, w_{g}}^{2} C \partial_{h, h}^{2} C-\left(\partial_{w_{g}, h}^{2} C\right)^{2}\right)<0
\end{aligned}
$$

and

$$
D_{4}=\left|\partial^{2} \mathcal{H}_{2}\right|=-\Theta " D_{3}>0
$$

It follows that the Hamiltonian is strictly concave for all $\left(p_{i}\right)_{i=1}^{2}$.

\section{Appendix C. Proof of proposition 2}

Let $\Phi:[0, R] \times[0, \bar{h}] \rightarrow R^{2}$ be defined by:

$$
\Phi\left(w_{g}, h\right)=\left(\begin{array}{c}
\Phi_{1}\left(w_{g}, h\right) \\
\Phi_{2}\left(w_{g}, h\right)
\end{array}\right)=\left(\begin{array}{c}
F^{\prime}(R)-\partial_{w_{g}} C\left(w_{g}, h\right)+\frac{1}{\rho} \partial_{h} C\left(w_{g}, h\right) \\
\Theta\left(\delta\left(R-\omega_{g}\right)\right)\left(\rho+\delta^{\prime}\left(R-\omega_{g}\right)\right)-\partial_{w_{g}} C\left(w_{g}, h\right)
\end{array}\right)
$$

and let us apply a standard degree theory argument (see for instance Mas-Colell, 1985, p.207-08). For that purpose, let us introduce $G\left(w_{g}, h\right)=\left(\begin{array}{c}h-A \\ B-w_{g}\end{array}\right)$ with $\left.(A, B) \in\right] 0, \bar{h}[\times] 0, R\left[\right.$. Obviously, $G\left(w_{g}, h\right)=0$ admits a unique solution given by $\left(w_{g}, h\right)=(B, A)$ and $\operatorname{det}\left(\left.\partial_{(w, h)} G\right|_{(B, A)}\right)=\left|\begin{array}{cc}0 & 1 \\ -1 & 0\end{array}\right|=1$, hence $\operatorname{deg}(G)=1$.

Let us now introduce the following homotopy $H\left(w_{g}, h, \lambda\right)=\lambda \cdot G\left(w_{g}, h\right)+(1-\lambda) \cdot \Phi\left(w_{g}, h\right)$, with $\lambda \in[0,1]$. A simple computation shows that:

$$
\forall \lambda>0, \quad \operatorname{rank}\left(\partial_{(A, B)} H\left(w_{g}, h, \lambda ;(A, B)\right)\right)=\operatorname{rank}\left(\left[\begin{array}{cc}
0 & -\lambda \\
\lambda & 0
\end{array}\right]\right)=2
$$

It follows by the generic transversality theorem (see Mas-colell, 1985, I22, p.45) that for almost all $(A, B) \in] 0, \bar{h}[\times] 0, R\left[\right.$, and $\lambda>0, \partial_{\left(w_{g}, h, \lambda\right)} H\left(w_{g}, h, \lambda ; A, B\right)$ is of full rank. So let us fix one of them. If we want to make sure that $H$ is a regular homotopy, we must verify that for $\lambda=0, \partial H\left(w_{g}, h, \lambda\right)$ is also of full rank. This is a simple exercise of computation, since:

$$
\begin{aligned}
\operatorname{det}\left(\partial_{w_{g}, h} H\left(w_{g}, h, 0\right)\right) & =\operatorname{det}(\partial \Phi)=\left[\begin{array}{cc}
\frac{1}{\rho} \partial_{h, w_{g}}^{2} C-\partial_{w_{g}, w_{g}}^{2} C & \frac{1}{\rho} \partial_{h, h}^{2} C-\partial_{w_{g}, h}^{2} C \\
\frac{d D}{d w_{g}}-\partial_{w_{g}, w_{g}}^{2} C & -\partial_{w_{g}, h}^{2} C
\end{array}\right] \\
& =\frac{1}{\rho}\left(\partial_{w_{g}, w_{g}}^{2} C \cdot \partial_{h, h}^{2} C-\left(\partial_{h, w_{g}}^{2} C\right)^{2}\right)-\frac{d D}{d w_{g}} \cdot\left(\frac{1}{\rho} \partial_{h, h}^{2} C-\partial_{w_{g}, h}^{2} C\right)
\end{aligned}
$$

with $D\left(w_{g}\right)=\Theta\left(\delta\left(R-\omega_{g}\right)\right)\left(\rho+\delta^{\prime}\left(R-\omega_{g}\right)\right)$. We deduce from our assumptions on the cost function 
(A2(i)(ii)) and the investment function (A3) that $\operatorname{det}(\partial \Phi)>0$. it follows that $\partial H\left(w_{g}, h, 0\right)$ is of full rank and more generally that the $H$ is a regular homotopy.

It follows from the classification theorem of 1-manifold that $H^{-1}(0)$ is diffeomorphic to segments or circles. Since "a segment starts at the regular solution of $G\left(w_{g}, h\right)=0$ ", it "reaches a solution of $\Phi\left(w_{g}, h\right)=0$ " if we verify that there exists no sequence

$$
\left(w_{g}^{n}, h^{n}, \lambda^{n}\right) \in H^{-1}(0) \text { and }\left(w_{g}^{n}, h^{n}, \lambda^{n}\right) \rightarrow\left(w_{g}^{0}, h^{0}, \lambda^{0}\right) \in(\{0, R\} \times\{0, \bar{h}\}) \times[0,1]
$$

If this condition is satisfied, the existence part of our proposition is proved. But we can go a step further. Since $\operatorname{det}(\partial \Phi)>0$, i.e $\Phi$ is a regular function, $\Phi^{-1}(0)$ only contains isolated points. It is moreover a closed subset of a compact set given by $[0, R] \times[0, \bar{h}]$, hence a finite set. By using a standard index theory argument (Mas-Colell 1985, J2 p46), we can say that $\sum_{\left(w_{g}, h\right) \in \Phi^{-1}(0)} \operatorname{ind}(\operatorname{det}(\partial \Phi))=1$ where $\operatorname{ind}(x)=1$ if $x>0$ and $\operatorname{ind}(x)=1$ if $x<0$. But the sign of $\operatorname{det}(\partial \Phi)$ is invariant. We can therefore also conclude that the solution to $\Phi\left(w_{g}, h\right)$ not only exists but is also unique.

To conclude the proof it therefore remains for us to verify that the boundary condition given in equation (C.1) is true. To do so, let us first observe that:

- Assumption A4(i) and (ii) and the fact that $\partial_{w_{g}} \Phi_{1}\left(w_{g}, h\right)<0$ bring us to the conclusion that:

$$
\left\{\begin{array}{c}
\forall w_{g} \in[0, R], \quad \Phi_{1}\left(w_{g}, 0\right)=F^{\prime}(R)-\partial_{w_{g}} C\left(w_{g}, 0\right)+\frac{1}{\rho} \partial_{h} C\left(w_{g}, 0\right)< \\
F^{\prime}(R)-\partial_{w_{g}} C(0,0)+\frac{1}{\rho} \partial_{h} C(0,0)<F^{\prime}(R)-\partial_{w_{g}} C(0,0)<0 \\
\forall w_{g} \in[0, R], \quad \Phi_{1}\left(w_{g}, \bar{h}\right)=F^{\prime}(R)-\partial_{w_{g}} C\left(w_{g}, \bar{h}\right)+\frac{1}{\rho} \partial_{h} C\left(w_{g}, \bar{h}\right) \\
>F^{\prime}(R)-\partial_{w_{g}} C(R, \bar{h})+\frac{1}{\rho} \partial_{h} C(R, \bar{h})>0
\end{array}\right.
$$

- By A4(ii) and the fact that $\partial_{d} \Phi_{2}\left(w_{g}, d\right)<0$, we can say that:

$$
\forall d \in[0, R], \Phi_{2}(0, d)=\Theta(\delta(R))\left(\rho+\delta^{\prime}(R)\right)-\partial_{w_{g}} C(0, d)>0
$$

- Finally, A3 and A2(ii) lead to:

$$
\forall d \in[0, R], \Phi_{2}(R, d)=-\partial_{w_{g}} C(R, d)<0
$$

It follows, from the first observation, that $\forall\left(w_{g}, \lambda\right) \in[0, R] \times[0,1]$

$$
\left\{\begin{array}{l}
H_{1}\left(w_{g}, 0, \lambda\right)=-\lambda d_{1}+(1-\lambda) \Phi_{1}\left(w_{g}, 0\right)<0 \\
H_{1}\left(w_{g}, \bar{d}, \lambda\right)=\lambda\left(\bar{d}-d_{1}\right)+(1-\lambda) \Phi_{1}\left(w_{g}, \bar{d}\right)>0
\end{array}\right.
$$

It is therefore impossible that there exists a sequence $\left(w_{g}^{n}, d^{n}, \lambda^{n}\right) \in H^{-1}(0)$ with $d^{n} \rightarrow d^{0} \in\{0, \bar{d}\}$. Let us now move to the the second and the third observations. We respectively conclude that $\forall(d, \lambda) \in$ 
$[0, \bar{d}] \times[0,1]$

$$
\left\{\begin{array}{l}
H_{2}(0, d, \lambda)=\lambda w_{1}+(1-\lambda) \Phi_{2}(0, d)>0 \\
H_{2}(R, d, \lambda)=\lambda\left(w_{1}-R\right)+(1-\lambda) \Phi_{2}(R, d)<0
\end{array}\right.
$$

Hence $\nexists\left(w_{g}^{n}, d^{n}, \lambda^{n}\right) \in H^{-1}(0)$ with $w_{g}^{n} \rightarrow w_{g}^{0} \in\{0, R\}$. We can therefore say that the boundary condition given in equation (C.1) holds.

\section{Appendix D. Proof of proposition 3}

We know from Dockner and Feichtinger (1991) that this system exhibits saddle-point stability with either real or conjugate complex root if (i) the determinant $D$ of this system is positive and (ii) $K=$ $M_{2}-r^{2}<0$ where $M_{2}$ stands for the sum of the second order principal minors of this system

Let us first verify that $K>0$. By computation, we have:

$$
\begin{aligned}
K & =\left|\begin{array}{cc}
-\frac{\partial_{w_{g}, h}^{2} C}{F^{\prime \prime}-\partial_{w_{g}, w_{g} C}^{2}} & -\frac{1}{F^{\prime \prime}-\partial_{w_{g}, w_{g} C}^{2} C} \\
\frac{\left(\partial_{w_{g}, h}^{2} C\right)^{2}}{F^{\prime \prime}-\partial_{w_{g}, w_{g}}^{2} C}+\partial_{h, h}^{2} C & \rho+\frac{\partial_{w_{g}, h}^{2} C}{F^{\prime \prime}-\partial_{w_{g}, w_{g}}^{2} C}
\end{array}\right|+\left|\begin{array}{cc}
-\delta^{\prime} & \frac{1}{\delta^{\prime \prime}} \\
p_{2} \cdot \delta^{\prime \prime}+\frac{F^{\prime \prime} \cdot \partial_{w_{g}, w_{g}}^{2} C}{F^{\prime \prime}-\partial_{w_{g}, w_{g} C}^{2} C} & \rho+\delta^{\prime}
\end{array}\right|+2\left|\begin{array}{cc}
\frac{\partial_{w_{g}, w_{g} C}^{2} C}{F^{\prime \prime}-\partial_{w_{g}, w_{g} C}^{2}} & 0 \\
-\frac{F^{\prime \prime} \cdot \partial_{w_{g}, h}^{2} C F^{\prime \prime}}{F^{\prime \prime}-\partial_{w_{g}, w_{g}}^{2} C} & 0
\end{array}\right| \\
& =-\frac{\rho \cdot \partial_{w_{g}, h}^{2} C}{F^{\prime \prime}-\partial_{w_{g}, w_{g}}^{2} C}+\frac{\partial_{h, h}^{2} C}{F^{\prime \prime}-\partial_{w_{g}, w_{g}}^{2} C}-\delta^{\prime} \cdot\left(\rho+\delta^{\prime}\right)-p_{2}-\frac{F^{\prime \prime} \cdot \partial_{w_{g}, w_{g}}^{2} C}{\delta^{\prime \prime} \cdot\left(F^{\prime \prime}-\partial_{w_{g}, w_{g}}^{2} C\right)}<0
\end{aligned}
$$

It remains fur us to verify that the determinant $D$ of this linearized system is positive. If we develop this expression with respect to the second line we can say that $D=D_{1}+D_{2}$ with:

$$
\begin{aligned}
D_{1} & =-\delta^{\prime} \cdot\left(\rho+\delta^{\prime}\right) \cdot\left|\begin{array}{cc}
-\frac{\partial_{w_{g}, h}^{2} C}{F^{\prime \prime}-\partial_{w_{g}, w_{g} C}^{2}} & -\frac{1}{F^{\prime \prime}-\partial_{w_{g}, w_{g} C}^{2}} \\
\frac{\left(\partial_{w_{g}, h}^{2} C\right)^{2}}{F^{\prime \prime}-\partial_{w_{g}, w_{g}} C}+\partial_{h, h}^{2} C & \rho+\frac{\partial_{w_{g}, h}^{2} C}{F^{\prime \prime}-\partial_{w_{g}, w_{g} C}^{2} C}
\end{array}\right| \\
& =\frac{-\delta^{\prime} \cdot\left(\rho+\delta^{\prime}\right)}{F^{\prime \prime}-\partial_{w_{g}, w_{g}}^{2} C} \cdot\left[\partial_{h, h}^{2} C-\rho \cdot \partial_{w_{g}, h}^{2} C\right]>0
\end{aligned}
$$

As the second term $D_{2}$ is more tedious to compute, we skip this computation and simply gives the result:

$$
\begin{aligned}
D_{2}= & \frac{1}{\delta "} \cdot\left[\begin{array}{ccc}
-\frac{\partial_{w_{g}, h}^{2} C}{F^{\prime \prime}-\partial_{w_{g}, w_{g}}^{2} C} & \frac{\partial_{w_{g}, w_{g} C}^{2} C}{F^{\prime \prime}-\partial_{w_{g}, w_{g} C}^{2} C} & -\frac{1}{F^{\prime \prime}-\partial_{w_{g}, w_{g} C}^{2} C} \\
\frac{\left(\partial_{w_{g}, h}^{2} C\right)^{2}}{F^{\prime \prime}-\partial_{w_{g}, w_{g}}^{2} C}+\partial_{h, h}^{2} C & -\frac{F^{\prime \prime} \cdot \partial_{w_{g}, h}^{2} C}{F^{\prime \prime}-\partial_{w_{g}, w_{g} C}^{2} C} & \rho+\frac{\partial_{w_{g}, h}^{2} C}{F^{\prime \prime}-\partial_{w_{g}, w_{g} C}^{2} C} \\
-\frac{F^{\prime \prime} \cdot \partial_{w_{g}, h}^{2} C}{F^{\prime \prime}-\partial_{w_{g}, w_{g} C}^{2} C} & p_{2} \cdot \delta^{\prime \prime}+\frac{F^{\prime \prime} \cdot \partial_{w_{g}, w_{g}}^{2} C}{F^{\prime \prime}-\partial_{w_{g}, w_{g}}^{2} C} & -\frac{\partial_{w_{g}, w_{g} C}^{2} C}{F^{\prime \prime}-\partial_{w_{g}, w_{g} C}^{2} C}
\end{array}\right] \\
= & \frac{p_{2} \cdot\left(\partial_{w_{g}, h}^{2} C \cdot \rho-\partial_{h, h}^{2} C\right)}{\left(F^{\prime \prime}-\partial_{w_{g}, w_{g}}^{2} C\right)}+\frac{\left(\partial_{w_{g}, h}^{2} C\right)^{2}-\partial_{w_{g}, w_{g}}^{2} C \cdot \partial_{h, h}^{2} C}{\delta^{\prime \prime} \cdot\left(F^{\prime \prime}-\partial_{w_{g}, w_{g}}^{2} C\right)}>0
\end{aligned}
$$




\section{References}

Amigues J-P., Favard P., Gaudet G., and Moreaux M., (1998), On the Optimal Order of Natural Resource Use when the Capacity of the Inhexhaustible Substitute is Limited, Journal of Economic Theory, 80, p.153-70.

Brovozovic N., Sunding D., Zilberman D., (2003), Optimal Management of Groundwater over Space and TIme, In : Berga D., Goetz R. (Eds), Frontier in Water Resource Economics, New York, Kluwer.

Dasgupta P.S. and Heal G.M., (1979), Economic Theory and Exhaustible Resources, Cambridge : Cambridge University Press.

Dockner E.J. and Feichtinger G., (1991) On the Optimality of Limit Cycles in Dynamic Economic Systems Journal of Economics 53 p 31-50

Fisher and Rubio, (1997), Adjusting to Climate Change : Implications of Increased Variability and Asymmetric Adjustment Costs for Investment in Water Reserves, Journal of Environmental Economics and Management, 34(3), p.207-227.

Gisser M., (1983), Groundwater : Focusing on the Real Issue, Journal of Political Economy, 91(6), p.1001-1027.

Gisser M. and Sanchez D., (1980), Competition versus Optimal Control in Groundwater Pumping, Water Resources Res., 16, p.638-642.

Grass D., Caulkins J., Feichtinger G., Tragler G. and Behrens D.A., (2008), Optimal Control of Non linear Processes with Applications to Drugs, Corruption and Terror, Springer Berlin, Heidelberg,

Hartl R. F.,Vickson R. and Sethi S.,(1995) A Survey of the Maximum Principles for Optimal Control Problems with State Constraints SIAM Review, Vol. 37,No. 2, pp. 181218 ,

Heal G., (1976), The Relationship between Price and Extraction Cost for a Resource with a Backstop Technology, Bell Journal of Economics, 7, p.371-378. 
Holland S.P. and Moore M.R., (2003), Cadillac Desert Revisited : Property Rights, Public Policy, and Water-Resource Depletion, Journal of Environmental Economics and Management, 46(1), p.131-155.

Knapp K and Olson L., (1995), The Economics of Conjunctive Groundwater Management with Stochastic Surface Supplies, Journal of Environmental Economics and Management, 28, p.340-356.

Kim C.S. and Moore M.R., (1989), Public Policies in Water-Resource Use : Their Effect on Groundwater Mining and Surface-Water Imports, Technical Bulletin 1764, U.S. Department of Agriculture, Economic Research Service.

Koundouri P., (2004), Current Issues in the Economics of Groundwater Resource Management, Journal of Economic Survey, 18(5), p.703-740.

Koundouri P. and Christou C., (2003), Dynamic adaptation to resource scarcity and backstop availability : Theory and Application to Groundwater, The Australian Journal of Agricultural and Resource Economics, 50, p.227-245.

Krulce D. L., Roumasset, J A., and Wilson, T.(1997), Optimal Management of a Renewable and Replaceable Resource: The Case of Coastal Groundwater, American Journal of Agricultural Economics, 79 (4) p.1218-28.

Lehmann C., Tsukada R. and Lourete A., (2010), Low-Cost TEchnologies Toward Achieving the Millenium Development Goals : The Case of Rainwater Harvesting, International Policy Centre for Inclusive Growth, 12, 4p.

Mas-Colell A. (1985), The Theory of General Economic Equilibrium : a Differentiable Approach, Cambridge University Press

Perrens S., (1982), Design Strategy for Domestic Rainwater Systems in Australia, Proc of 1st International Conference on Rainwater Cistern Systems, Hawaii, Honolulu.

Provencher B., (1993), A private property rights regime to replenish a groundwater aquifer, Land Economics; 69(4) p.325-341. 
Provencher B. and Burt O., (1993), The Externalities associated with the Common Property Exploitation of Groundwater, Journal of Environmental Economics and Management, 24, p.139-158.

Provencher B., (1995), Issues in the Conjunctive Use of Surface Water and Groundwater, In D. Bromley, ed. Handbook of Environmental Economics, Blackwell.

Rubio S.J. and Casino B., (2003), Strategic Behavior and Efficiency in the Common Property Extraction of Groundwater, Environmental and Resource Economics, 26(1) p.7387.

Saak A. and Peterson J., (2007), Groundwater Use under Incomplete Information, Journal of Environmental Economics and Management, 54, p.214-228.

Seierstad A. and Sydsaeter K., (1987), Chap.6 : Mixed and Pure State Constraints, In : Optimal Control Theory with Economic Applications, North-Holland Publisher.

Stahn H. and Tomini A., (2010) A drop of Rainwater against a drop of Groundwater : Does Rainwater Harvesting allows us really to spare groundwater? GREQAM Working Paper.

Tsur Y., (1990), The Stabilization Role of Groundwater when Surface Water Supplies are Uncertain : The Implications for Groundwater Development, Water Resources Research, 26(5), p.811-818.

Tsur Y. and Graham-Tomasi T., (1991), The Buffer Value of Groundwater with Stochastic Surface Water Supplies, Journal of Environmental Economics and Management, 21, p.201224 . 